\title{
SOBRE A INTERPRETAÇÃO FREQUENTISTA DE PROBABILIDADE
}

\author{
André Neiva \\ Doutorando em Filosofia na PUCRS \\ al.neiva@gmail.com
}

Resumo: Neste breve artigo, forneço um exame crítico da teoria frequentista de probabilidade, que geralmente se apresenta de duas formas diferentes: frequentismo atual e frequentismo hipotético. Considero a interpretação frequentista como uma forma de reducionismo, uma vez que pretende explicar probabilidades físicas em termos de frequências relativas (em uma classe de referência atual ou hipotética). Também discuto algumas das principais objeções contra o frequentismo atual e hipotético e avalio se tais teorias estão de acordo com um conjunto de critérios de adequação para interpretações de probabilidade física. No final, concluo que o frequentismo não oferece uma análise satisfatória do conceito de probabilidade física.

Palavras-chave: Frequentismo. Probabilidade Física. Teoria de Probabilidade.

\section{ON THE FREQUENTIST INTERPRETATION OF PROBABILITY}

Abstract: In this brief paper, I provide a critical examination of the frequency theory of probability, which generally comes in two different forms: actual frequentism and hypothetical frequentism. I regard the frequency interpretation as a form of reductionism, since it attempts to explain physical probabilities in terms of relative frequencies (either in an actual or hypothetical reference class). I also discuss some of the main objections against both actual and hypothetical frequentism, and I examine whether or not those theories are in accordance with a set of criteria of adequacy for the interpretations of physical probabilities. In the end, I conclude that frequentism does not offer a satisfactory analysis of the concept of physical probability.

Keywords: Frequentism. Physical Probability. Probability Theory.

\section{Introdução}

Diferentes concepções e interpretações de probabilidade têm sido propostas ao longo da história. Apesar disso, a teoria matemática de probabilidade é relativamente recente. É somente no século XX que a teoria recebe uma formulação 
precisa com o trabalho Fundamentos da Teoria de Probabilidade (1956 [1933]) do matemático russo A. N. Kolmogorov. O maquinário formal desenvolvido por Kolmogorov, no entanto, parece ser neutro em relação à interpretação dos termos 'provável' e 'provavelmente', assim como não nos diz nada sobre a existência de diferentes tipos de probabilidade.

Neste artigo, apresento e examino uma interpretação específica de probabilidade: o frequentismo. O propósito do trabalho é dar um acesso informativo e crítico ao debate que envolve esse tipo de teoria. Em geral, o frequentismo acerca da probabilidade é dividido em dois grandes grupos, nomeadamente, o frequentismo atual e o frequentismo hipotético. Após fornecer algumas distinções e estabelecer um conjunto mínimo de desideratos, exploro em mais detalhes no que consiste tais teorias. Considero a sua versão mais forte: o frequentismo como uma análise do conceito de probabilidade física (ou natural). Também apresento alguns dos principais problemas que ambas formas de frequentismo enfrentam. A sugestão é a de que frequentistas falham na sua tarefa de proporcionar uma análise apropriada de tal conceito.

\section{Tipos de Probabilidade}

É conveniente distinguir entre dois tipos gerais de probabilidade: um epistêmico e um físico. ${ }^{1} \mathrm{O}$ primeiro deles diz respeito à relação de confirmação entre um conjunto de proposições evidenciais e uma dada hipótese, a saber, determina em que grau um conjunto de evidências $E$ probabiliza uma hipótese $H$. Nessa perspectiva, probabilidades epistêmicas são sempre relativas a um corpo evidencial. Essa é a sua marca distintiva. As afirmações abaixo representam dois dos seus exemplos paradigmáticos:

(1) Assumindo as evidências astronômicas disponíveis, é provável que a Teoria do Big Bang seja verdadeira;

(2) Novas evidências tornam provável a hipótese de que Suzane cometeu um crime.

Ao passo que probabilidades epistêmicas medem o grau de confirmação que evidências fornecem a hipóteses, probabilidades físicas tipicamente descrevem certas características ou aspectos do mundo. Assim, a sua natureza não parece repousar em qualquer fato acerca do que sabemos, acreditamos, esperamos ou se alguma vez pensamos a seu respeito. Ou seja, a sua existência é tomada como independente do que se passa na nossa vida mental. As seguintes afirmações constituem casos de probabilidades físicas:

(3) É tão provável obter o resultado coroa quanto cara ao lançar uma moeda;

(4) É provável que um átomo de rádio sofra decaimento radioativo dentro de 1600 anos.

\footnotetext{
${ }^{1}$ Aqui segue uma pequena advertência. O rótulo 'físico' não implica que esse tipo de probabilidade figura somente em teorias físicas. Presumivelmente, probabilidades físicas também aparecem em outras áreas da ciência, como a biologia e a psicologia. Além disso, importa dizer que a distinção acima é sugerida por Ramsey (1950 [1926]) e Carnap (1962 [1950]), embora o primeiro contraste probabilidade física com probabilidade subjetiva (o grau de crença de um agente) e o segundo a contraponha com o grau de confirmação, sendo que este é dado por probabilidades lógicas.
} 
Os exemplos acima ajudam a marcar a diferença entre os dois tipos de probabilidade. Enquanto um diz respeito a uma relação de suporte que evidências conferem a hipóteses, o outro corresponde a eventos e fatos do mundo. Vou me concentrar no segundo tipo de probabilidade. Mais precisamente, o objeto deste artigo é o frequentismo enquanto uma teoria de probabilidade física, não epistêmica. ${ }^{2}$ Talvez no que seja a sua pretensão mais forte, o frequentismo promove uma análise reducionista: probabilidades físicas são frequências relativas. Essa será a maneira pela qual vou entender os dois projetos frequentistas. ${ }^{3}$

Filósofos normalmente assumem que proposições ou sentenças são o objeto ao qual se anexa os termos 'provável' e 'provavelmente'. Porém, quando falamos de probabilidades físicas, parece mais natural considerar eventos como o seu portador. ${ }^{4}$ Não quero entrar nessa disputa, que às vezes nos desvia do ponto mais fundamental em jogo. De qualquer forma, é importante notar que é possível traduzir a fala de eventos para a fala em termos de proposições. Observe as declarações abaixo, que são convertíveis entre si:

(†) Um evento $A$ de um tipo a tem probabilidade física $x$ de ocorrer;

( ) A probabilidade física de uma proposição $P$ é $x$, onde $P$ diz que um evento $A$ de um tipo a ocorre. ${ }^{5}$

Desse modo, não há nenhum grande prejuízo para a discussão a ser realizada se o leitor preferir adotar a posição da ortodoxia quanto aos portadores de probabilidades.

\subsection{Critérios de Adequação}

É justo perguntar quais critérios de adequação interpretações de probabilidade devem satisfazer. À luz de um conjunto apropriado de critérios, teorias de probabilidade física seriam avaliadas e, em última instância, receberiam um veredito mais claro quanto à interpretação pretendida. Ainda que brevemente, Salmon (1966, p. 63-65) e Hájek (2012, seção 2) perseguem essa via, embora não apresentem um grupo específico de critérios para interpretações de probabilidade física. Forneço abaixo alguns desideratos que, em certa medida, coincidem com alguns dos seus critérios. Mesmo que cada um deles me pareça ser individualmente necessário, talvez não constituam critérios conjuntamente suficientes de adequação.

Além de explicar o que são probabilidades físicas, parece correto exigir que interpretações cumpram com os seguintes desideratos:

\footnotetext{
2 Para uma discussão de como a noção de confirmação poderia ser explicada por frequências relativas e dos principais problemas relacionados a essa visão, ver Achinstein (2001, p. 52-58).

${ }^{3} \mathrm{~A}$ interpretação frequentista de probabilidade discutida neste artigo não deve ser confundida com a abordagem frequentista ou inferência frequentista em estatística. Esta, diferentemente, estuda diferentes métodos e ferramentas para testar hipóteses, como (por exemplo) o teste de hipóteses ou teste de significância pelo uso do valor- $p$.

${ }_{4}^{4}$ Em teoria de probabilidade, um evento é formalmente definido como qualquer subconjunto do espaço de amostra, isto é, um subconjunto do conjunto de todos resultados possíveis de um experimento. Assim, não somente eventos atuais devem ser levados em conta, mas também eventos possíveis. Para mais informações sobre os portadores de probabilidade, ver Handfield (2012, cap. 1) e Hájek \& Hitchcock (2016, subseção 1.8).

${ }^{5}$ Handfield (2012, p. 5) chama igualmente atenção para essa tradução.
} 
(a) Permitir que probabilidades físicas sejam descobertas, tornando valores não-extremos ( $\neq$ de 1 e 0 ) uma possibilidade;

(b) Ser aplicável à ciência, identificando claramente o seu emprego, e ser compatível com as teorias científicas contemporâneas mais bem-sucedidas.

O primeiro é em parte o que Salmon chama de 'verificabilidade' em combinação com o que Hájek chama de 'não-trivialidade'. Por isso, (a) pode ser visto como um grupo de desideratos. Formalmente, uma função de probabilidade assinala valores reais do intervalo $[0,1]$ aos seus portadores, nomeadamente, a eventos. Qualquer valor distinto de 0 e 1 é um valor não-extremo ou intermediário. Note que existem incontáveis números irracionais neste intervalo. Além disso, uma interpretação seria insatisfatória se tornasse obscuro o modo pelo qual se descobrem probabilidades. Nesse sentido, (a) determina que não se exclua uma distribuição que assinala qualquer um desses números irracionais e que seja possível descobrir tais probabilidades por meio de algum método. O segundo é o que Hájek designa por 'aplicabilidade à ciência'. Incluo a cláusula de compatibilidade com o que há de melhor na ciência, que não é explicitamente formulada por ele. Dificilmente consideraríamos como correta uma interpretação incompatível com o que as teorias mais bem-sucedidas nos dizem sobre o mundo.

Parece-me que (a) e (b), a despeito do minimalismo, constituem critérios aos quais interpretações de probabilidade física devem obedecer. Penso que é uma reivindicação legítima examinar como probabilidades físicas devem ser modeladas formalmente, isto é, qual versão do cálculo de probabilidades é mais adequada a tal tipo de entidade. Isso nos levaria a uma discussão mais técnica e complicada. Afinal, o cálculo de Kolmogorov é somente uma alternativa, usualmente apresentado como - modelo padrão, onde probabilidade categórica é primitiva. ${ }^{6}$ Não farei essa exigência, deixando essa questão em aberto. O importante é ter em mente (a) e (b) quando ambas formas de frequentismo forem avaliadas.

\section{Frequentismo Atual}

Não é um exagero afirmar que o frequentismo encontra muitas das suas raízes em uma visão de senso comum em torno do conceito de probabilidade. As pessoas costumam pensar que probabilidade é simplesmente a proporção na qual um evento ocorre: o número de instâncias favoráveis pelo número total de instâncias de um experimento. Exemplos em contextos de jogos de azar e apostas fortalecem esse apelo intuitivo. Por sua vez, a ciência está permeada por alguma versão de teoria frequentista, principalmente devido à ideia de frequência relativa na qual um tipo evento ocorre em uma série de experimentos de longo prazo. ${ }^{7}$ Manuais de estatística e ciências evidenciam esse comprometimento.

\footnotetext{
6 Tal como Hájek (2003) defende, talvez existam boas razões para inverter a ordem de análise assumida pelo maquinário de Kolmogorov. Em vez de probabilidade categórica, probabilidade condicional se tornaria a noção primitiva e a primeira seria definida em termos da segunda. A mesma neutralidade que mencionei no início permaneceria em um cálculo de probabilidades com esse formato.

${ }^{7}$ Para um estudo dos padrões subjacentes à inferência estatística e da noção de frequência de longo prazo, ver lan Hacking (1965), certamente um dos trabalhos mais influentes sobre o assunto.
} 
A versão mais simples de teoria frequentista iguala probabilidades a frequências relativas atuais. A análise do frequentismo atual é articulada da seguinte maneira: ${ }^{8}$

(FA) A probabilidade de um evento $A$ em uma classe de referência $X$ é a frequência relativa na qual $A$ atualmente ocorre em $X^{9}$

Frequentistas entendem que probabilidades devem ser sempre relativas a uma classe de eventos, isto é, a uma classe de referência. Uma classe corresponde a todos os resultados de um determinado tipo de evento. A princípio, pode ser finita ou infinita. Os exemplos aqui serão com classe finita, embora na próxima seção eu exponha os problemas que (FA) se defronta ao permitir classes infinitas.

No caso de (FA), as ocorrências atuais de $A$ em relação a uma classe de referência $X$ vão constituir a frequência relativa observada de $A$ em $X$. Essa frequência é dada por uma razão $\frac{m}{n}$, onde $m$ é o número de ocorrências de $A$ e $n$ é o número total de ocorrências dos resultados que formam $X$. Por exemplo, suponha que um mecanismo lança repetidamente um dado de seis lados. Os resultados que podem sair a cada jogada formam o conjunto $\Omega=\{1,2,3,4,5,6\}$. A frequência relativa do evento $\{2\}$ é a proporção de vezes que tal resultado é obtido pelo número total de ocorrências em que os resultados de $\Omega$ são obtidos. Agora um exemplo mais científico. Imagine que de uma amostra de 10000 homens fumantes na faixa de 55 a 60 anos 4500 tenham algum tipo de câncer. Suponha que a amostra represente bem a população sobre a qual uma pesquisa deve ser aplicada. Assim, a frequência relativa é simplesmente a razão entre a cardinalidade do conjunto de homens fumantes com câncer pela cardinalidade do conjunto de homens fumantes. A frequência observada de câncer em homens na amostra (fumantes entre 55 a 60 anos) é de $45 \%$ e, evidentemente, a sua probabilidade é 0.45 de acordo com (FA).

Ainda que o frequentismo atual e a interpretação clássica de probabilidades possuam algumas semelhanças, existem divergências cruciais entre as duas abordagens. Na interpretação clássica, onde Pierre-Simon Laplace (1951 [1814]) é o seu principal representante, probabilidades são concebidas como medidas de possibilidade: a probabilidade é a razão entre o número de casos favoráveis pelo número total de casos possíveis, sendo que tais possibilidades pertencem ao espaço de amostra considerado. O frequentista atual, ao contrário, limita a sua análise meramente aos resultados atuais de um experimento. Portanto, a frequência é definida como a proporção das instâncias favoráveis pelo número total de instâncias que de fato ocorrem.

Geralmente o frequentismo atual é associado a uma visão Humeana quanto à natureza da causação. ${ }^{10}$ De acordo com essa concepção, a causação entre eventos é explicada como uma sucessão de eventos regulares, mas sem introduzir qualquer conexão necessária entre os relata causais. Na verdade, dizemos que um evento $A$

$8 \mathrm{O}$ frequentismo atual não é propriamente uma posição popular entre filósofos. Não raramente John Venn (1888 [1866]) é visto como um dos precursores de tal concepção, ao defender que probabilidade é meramente a proporção de indivíduos ou atributos em uma determinada classe. Para uma visão geral, ver Hájek (2012, subseção 3.4).

9 Vale enfatizar que (FA) é em muitos aspectos semelhante à formulação sugerida por Hájek (1997, p. 212) e La Caze (2016, p. 342). A diferença relevante é que, em vez de eventos, esses autores utilizam atributos ou propriedades como os portadores de probabilidades.

10 Para mais informações, ver Mellor (2005, cap. 3) e Bradley (2015, cap. 9). 
de um tipo a causa um evento $B$ de um tipo $\beta$ porque eventos- $\alpha$ e eventos- $\beta$ costumam estar em uma relação de conjunção constante. Conforme Mellor (2005, p. 39) chama atenção, ambas posições combinadas oferecem uma análise a partir da qual noções modais são substituídas por noções não-modais. Para Mellor, isso permite lidar com as noções de causação e necessidade nômica. ${ }^{11} \mathrm{Em}$ linhas gerais, a substituição equivale a:

\section{'A é necessário' passa a ser 'A sempre ocorre';}

'A é possível passa a ser ' $A$ às vezes ocorre'.

Por consequência, o que é impossível passa a ser o que nunca ocorre. 0 vocabulário modal é então substituído por um vocabulário frequentista. De fato, é bem questionável se tais primitivos não-modais conseguem desempenhar papel explanatório similar ao papel que modalidades antes exerciam. Seja como for, o ponto relevante aqui é que essa explicação alternativa do frequentista nos revela mais um contraste entre a sua proposta e a interpretação clássica. Enquanto a segunda preserva modalidades, a primeira simplesmente as elimina.

\subsection{Problemas para o Frequentismo Atual}

Apesar de intuitiva e simples, a abordagem proposta pelo frequentista atual possui sérios problemas. Nesta seção, discuto algumas das objeções centrais a essa posição. ${ }^{12}$

Em primeiro lugar, teorias frequentistas precisam lidar com o problema da classe de referência. Parece haver diferentes classes de referência às quais probabilidades pertencem. Usando o exemplo de Hájek (1997, p. 74), suponha que eu queira saber qual é a probabilidade da minha morte aos 60 anos. Existe a classe de todos os homens, mas também a de todos os homens não fumantes, a de todos os homens brasileiros não fumantes e assim por diante. Não há garantia que para cada uma dessas classes existe uma única frequência relativa e, portanto, um único valor de probabilidade física de que eu morra aos 60 anos. Qual delas deve-se escolher? Reichenbach (1949, p. 374) pensa que a classe de referência com menor escopo para a qual existe estatística confiável deve ser privilegiada. Ora, isso parece funcionar quando, em primeiro lugar, há estatística confiável e, em segundo lugar, quando uma classe é uma subclasse de uma outra classe. Por exemplo, a classe de homens brasileiros não fumantes é uma subclasse da classe de homens não fumantes. Se houvesse estatística confiável para ambas, a primeira deveria ser privilegiada sobre a última. Contudo, isso nos dá somente um critério parcial de decisão. Afinal, o problema ainda persiste para classes de mesmo escopo, que não são subclasses uma da outra, para as quais existe estatística confiável.

11 O defensor da visão Humeana ainda poderia alegar que as leis da natureza são apenas um subconjunto de regularidades acidentais. É claro que uma reação comum seria argumentar que regularidades acidentais não podem constituir uma lei natural, uma vez que são espúrias. Embora interessante, esse debate se situa além do propósito deste texto. O importante nesta altura é demarcar a diferença entre as duas interpretações, a frequentista e a clássica.

12 Ao leitor interessado, ver Hájek (1997). No seu artigo, Hájek apresenta diversos argumentos contra o frequentismo atual. Julgo que a grande maioria dos seus argumentos é bastante contundente. Alguns são mais técnicos e outros mais intuitivos. Restrinjo-me a alguns dos mais populares. 
Como disse anteriormente, o valor de uma frequência relativa é uma proporção $\frac{m}{n}$, onde $m$ e $n$ são números inteiros não negativos. Para o frequentista atual, probabilidades invariavelmente terão esse formato. No entanto, lembre-se que existem muitos números irracionais, incontáveis deles, no intervalo [0,1]. Por exemplo, o número irracional $\frac{1}{\sqrt{5}}$ se situa em $[0,1]$. Por que eliminar essa possibilidade? A análise em (FA) exclui a possibilidade de assinalar probabilidades irracionais a eventos. Muitas teorias científicas, como a mecânica quântica, nos dizem que existem probabilidades irracionais. ${ }^{13}$ Assim, (FA) falha em satisfazer 0 desiderato (b) de aplicabilidade à ciência. Embora não permita valores irracionais, (FA) cumpre com o grupo (a) de desideratos. Valores não-extremos são possíveis e a probabilidade de um tipo de evento pode ser descoberta pela observação da frequência relativa atual correspondente.

Falei até agora de classes finitas. Mas o que acontece se assumirmos a possibilidade da existência de classes infinitas? As consequências não são muito promissoras para (FA). Por um lado, não haveria frequência relativa no caso de ocorrências infinitas de um tipo de evento em uma sequência infinita de resultados: $\infty$ não é determinado. Por outro lado, a frequência relativa seria 0 no caso de ocorrências finitas de um tipo de evento em uma sequência infinita de resultados: $a_{/ \infty}=0$ para qualquer $a \in \mathbb{R}$, onde $-\infty<a<\infty$. Nenhuma delas favorece a análise do conceito de probabilidade física em termos de frequências atuais em classes infinitas.

\section{Frequentismo Hipotético}

Uma versão mais refinada de teoria frequentista iguala probabilidade física ao limite de uma frequência relativa hipotética. Reichenbach (1949) e von Mises (1957 [1928]) estão entre os seus principais proponentes. Tais autores aceitam classes de referência infinitas e apelam à noção matemática de limite. Nessa visão, a ideia de frequência relativa de um tipo de evento em uma sequência de experimentos repetidos de longo prazo recebe um tratamento mais sofisticado. ${ }^{14}$

Note que a expressão 'sequência de longo prazo' é vaga. Em virtude disso, frequentistas hipotéticos endossam a maior sequência possível e defendem que a análise seja em termos contrafactuais: o que teria acontecido se uma sequência de resultados fosse infinita. A tese é de que a probabilidade seja igual ao que teria sido o limite da frequência relativa se uma sequência tivesse uma amplitude infinita. Mais precisamente, o frequentismo hipotético equivale a:

(FH) A probabilidade de um evento $A$ em uma classe de referência $X$ é o limite da frequência relativa na qual $A$ teria ocorrido em relação a $X$ caso $X$ fosse estendida infinitamente. ${ }^{15}$

\footnotetext{
13 Importa enfatizar que, como Hájek (1997, p. 84) bem observa, qualquer versão de frequentismo também elimina a possibilidade de probabilidades infinitesimais.

14 Para uma visão geral, ver Kyburg Jr. \& Teng (2001, cap. 4) e Hájek (2012, subseção 3.4).

15 Mais uma vez, a formulação (FH) que forneço é muito semelhante às sugestões de Hájek (2009, p. 212) e La Caze (2016, p. 346); estes entendem que atributos ou propriedades são os portadores de probabilidades.
} 
No intuito de tornar $(\mathrm{FH})$ clara o suficiente, será útil entender no que consiste a noção de limite de uma sequência. Para tanto, considere a sequência de números:

$$
\frac{1}{1}, \frac{1}{2}, \frac{1}{3}, \cdots, \frac{1}{n}, \cdots
$$

Imagine que cada um dos números está em uma posição: $1 / 1$ está na posição um, $1 / 2$ na posição dois e assim por diante. O número $n$ se torna cada vez maior à medida que a sequência é percorrida. Dessa maneira, o próximo número $1 / n$ na próxima posição está mais perto de um limite $L$. Por assim dizer, a distância para $L$ se torna cada vez menor. Neste caso, $L=0$. Significa que $1 / n \rightarrow 0$ quando $n \rightarrow \infty$. Formalmente, para qualquer $x \in \mathbb{R}_{>0}$, há uma posição $\zeta$ tal que qualquer número depois de $\zeta$ é diferente de 0 por menos de $x$. Eis uma definição mais rigorosa: para qualquer $x \in \mathbb{R}>0$, há um $m \in \mathbb{N}$ tal que para qualquer $n \in \mathbb{N}$, onde $n \geq m,\left|S_{n}-L\right|<x$. Ou seja, $\lim _{n \rightarrow \infty} S_{n}=L$. Quando uma sequência $\left\{S_{n}\right\}$ tende a um limite, $\left\{S_{n}\right\}$ é convergente. Quando $\left\{S_{n}\right\}$ não tende a um limite, $\left\{S_{n}\right\}$ é divergente.

Agora nos cabe aplicar o conceito de limite a frequências relativas. A esse respeito, o exemplo formulado por Mellor (2005, p. 42) é ilustrativo. Suponha uma sequência de frequências relativas em que $n$ átomos de rádio sofrem decaimento radioativo no intervalo de 1000 anos. Veja a tabela 1 a seguir:

Tabela 1: Sequência de Frequências

\begin{tabular}{|l|l|l|l|l|}
\hline 10 & 100 & 1000 & 10000 & $\ldots$ \\
\hline 2 & 34 & 301 & 3145 & $\ldots$ \\
\hline 0.2 & 0.34 & 0.301 & 0.3145 & $\ldots$ \\
\hline
\end{tabular}

A primeira e a segunda linhas exibem, respectivamente, o número total de átomos de rádio e o número daqueles átomos que efetivamente sofrem decaimento no período referido. Já a terceira linha mostra as frequências relativas de cada um dos períodos. As colunas representam diferentes posições, sendo que cada uma se refere a um intervalo de 1000 anos. Assumindo que existe um limite $L$, onde $L \in$ $[0,1]$, o raciocínio é semelhante ao exemplo da sequência de números. A frequência relativa subsequente, situada na próxima posição, tenderá cada vez mais ao limite $L$. Assim, $L$ é o limite da frequência relativa de átomos de rádio decaídos. Sob a suposição de que a sequência é estendida infinitamente, $(\mathrm{FH})$ afirma que $L$ corresponderá à probabilidade física de um átomo entrar em decaimento radioativo em 1000 anos.

Ainda que seja uma caracterização mais geral do frequentismo hipotético, o que foi exposto acima preserva o que há de comum nas teorias de von Mises e Reichenbach. Na próxima seção, ao tratar de um problema específico, mostrarei como a teoria pode ser fortalecida pelo acréscimo de duas condições. Focarei na proposta de von Mises. 


\subsection{Problemas para o Frequentismo Hipotético}

A análise do frequentista hipotético não está imune a problemas. Existem vários bons argumentos contra a sua teoria. Por questão de espaço, vou explorar em detalhes um deles e discutirei os desideratos da seção 2 no final. ${ }^{16}$

O limite de uma sequência pode ser diferente se a sua ordem for alterada. Considere 0 conjunto dos números naturais $\mathbb{N}>0$, ou seja, $\mathbb{N}_{>0}=\{x \in \mathbb{N} \mid x \neq 0\}$. Na ordem regular, o limite da frequência na qual extraímos um número par de $\mathbb{N}>0$ é $1 / 2$ :

$$
\frac{1}{2}, \frac{1}{3}, \frac{2}{4}, \frac{2}{5}, \frac{3}{6}, \frac{3}{7}, \frac{4}{8}, \frac{4}{9}, \frac{5}{10}, \cdots
$$

Quanto mais números obtemos do conjunto $\mathbb{N}>0$, mais o valor da frequência relativa de se conseguir um número par converge para $\frac{1}{2}$. Se, por acaso, a ordem dos números em $\mathbb{N}_{>0}$ for modificada, o limite poderá ter outro valor. Por exemplo, a frequência na qual se obtém números pares de $3,5,7,9,2,11,13,1,15,4, \ldots$ tenderia ao limite $\frac{1}{5}$. Por conseguinte, o limite da frequência relativa de um tipo de evento em uma classe está sujeito a uma dada ordem. Em outras palavras, esse caso simples nos mostra que o limite da frequência pode sofrer alteração quando a ordem for diferente. Significa que, para (FH), a probabilidade igualmente dependerá da ordem na qual os resultados de um experimento são obtidos.

O problema da ordem tem alguns efeitos importantes. Um deles, que é apontado por Hájek (2009, p. 219), consiste na introdução de um novo elemento na análise do frequentista hipotético. Mesmo que se tenha um critério razoável para escolher a classe de referência relevante, a relação agora é de três lugares, uma vez que temos a inclusão de uma determinada ordem: 'o limite da frequência relativa é $L$, dada uma classe $X$ e uma ordem $O$ '. A pergunta natural a se fazer ao frequentista é: por que probabilidades físicas precisam depender da ordem dos resultados? Está longe de ser claro por que razão se deve incluir essa condição. No final das contas, o frequentista ainda precisaria de um critério para estabelecer qual ordem deve ser favorecida.

Frequentistas como von Mises (1957 [1928], p. 24-25) sugerem que existe uma maneira apropriada na qual podemos restringir sequências infinitas. Estas devem satisfazer certas condições que as tornem bem formadas ou definidas. Tais sequências são conhecidas como collectives. Frequências relativas hipotéticas são definidas somente se pertencem a tal tipo de sequência. Dado que $S$ é uma sequência enumeravelmente infinita, as condições exigidas são: ${ }^{17}$

(i) Há um limite da frequência relativa para qualquer evento de $S$;

\footnotetext{
${ }^{16}$ Ao leitor interessado, ver Hájek (2009). Na continuação do seu artigo de 1997, Hájek oferece mais quinze argumentos contra o frequentismo, mas agora direcionados à versão hipotética.

17 Para mais informações a respeito das condições, ver Gillies (2000, cap. 5) e Kyburg Jr. \& Teng (2001, cap. 4).
} 
(ii) O limite da frequência relativa de qualquer evento em $S$ é igual ao seu limite em qualquer subsequência infinita de $S$, onde uma subsequência é gerada por um método recursivo.

O primeiro critério é o de convergência, já que exige a existência de um limite. Por exemplo, não há o limite da frequência de resultados coroa quando um mecanismo lança uma moeda e obtemos séries de $2^{n}$ resultados de coroa seguidas por séries de $2^{n}$ resultados de cara. O segundo critério é o de aleatoriedade. Em linhas gerais, essa condição torna impossível a existência de qualquer sistema bemsucedido de apostas a partir do qual uma pessoa poderia predizer os resultados e garantir a sua vitória. Por exemplo, uma sequência alternada de resultados cara e coroa não é considerada randômica. Dessa forma, qualquer um que aposte em coroa em todas instâncias pares de tal sequência teria ganho garantido. Subsequências de uma sequência infinita devem preservar o limite da frequência. ${ }^{18}$ Uma subsequência é produzida por um método no qual seleciona a posição dos resultados que constituem a sequência. Quando a seleção da posição de um resultado altera o limite da frequência na subsequência, a sequência original não é considerada um collective e a probabilidade não é definida. Em último caso, o frequentista pode alegar que probabilidades não são definidas se a sequência envolvida não tem um limite ou se os seus resultados são predizíveis por um sistema bem-sucedido de apostas.

Ambos critérios permitem que o frequentista elimine várias sequências espúrias da sua análise. Embora isso responda parcialmente ao problema da ordem, existem outros problemas em relação ao critério (ii) de aleatoriedade. Hájek (2009, p. 224-225) identifica dois deles. Primeiro, a condição é restritiva, uma vez que exclui processos e sequências que em geral parecem ser randômicas. Um mecanismo honesto que lança uma moeda é um processo dessa natureza, quaisquer que sejam os seus resultados; se engendram ou não uma sequência tal como um collective. Segundo, a condição é muito ampla. Uma sequência com uma sucessão de resultados coroa, sem um único resultado cara, cumpre com a condição (ii). O limite da sua frequência é 1 na sequência infinita e em quaisquer das suas subsequências. Entretanto, dificilmente diríamos que se trata de uma sequência produzida por um processo randômico. Intuitivamente, uma sequência na qual o mesmo resultado é obtido em todas as instâncias de um experimento não parece ser gerada aleatoriamente.

Por último, devemos aplicar os desideratos de adequação sobre (FH). O critério (a) é parcialmente satisfeito. De fato, o limite de uma frequência pode assumir qualquer número real de $[0,1]$. Não há nenhuma restrição em relação a probabilidades irracionais. Porém, não é claro como se deve fixar frequências hipotéticas. Devemos lembrar que análise em $(\mathrm{FH})$ é formulada em termos contrafactuais. Neste caso, o frequentista hipotético poderia alegar que o limite da frequência deve corresponder a um valor correto nos mundos possíveis mais próximos onde sequências infinitas ocorrem, assumindo que contrafactuais são explicados pela semântica tradicional de mundos possíveis. O ponto é que não há nenhuma garantia disso. Por exemplo, se um mecanismo honesto lança uma moeda 100000 vezes e obtém-se 38000 resultados coroa, a sua frequência é igual a 0.38. Imagine que essa seja efetivamente a sua frequência no mundo atual. Entretanto,

${ }_{18}$ Cabe notar que essa ideia foi aperfeiçoada por Church (1940) ao defini-la como uma função recursiva. 
por ser um mecanismo honesto, o frequentista afirmaria que, nos mundos mais próximos em que a moeda é lançada infinitamente, o limite da frequência do resultado coroa deve corresponder a $\frac{1}{2}$, que consistiria no seu valor correto, e não a 0.38. Ora, por que isso seria o caso nos mundos possíveis mais próximos em que a sequência de resultados tem uma amplitude infinita se no mundo atual a frequência relativa é 0.38 ? Como Hájek (2009, p. 222) salienta, isso representa um afastamento arbitrário do que acontece no mundo atual.

Quanto ao critério (b), não é claro a utilidade e aplicabilidade de frequências hipotéticas, mesmo que o limite de uma frequência possa ter aplicações na ciência. Diferentemente, o emprego de frequências atuais é expressivo na pesquisa e áreas científicas.

\section{Considerações Finais}

As duas versões de frequentismo oferecem uma explicação reducionista de probabilidades: estas são frequências relativas. Ambas posições, contudo, estão sujeitas a sérios problemas. Enquanto o frequentista atual elimina a possibilidade de probabilidades irracionais e precisa lidar com o problema da classe de referência, o problema da ordem e a condição de aleatoriedade causam dificuldades para o frequentista hipotético. Apesar disso, frequências relativas ainda são um bom guia para descobrir probabilidades no mundo. Com efeito, existe uma relação íntima entre frequências relativas e probabilidades - a noção de estabilidade estatística e os teoremas do limite são representativos a esse respeito -, mas elas não parecem ser o mesmo tipo de entidade. Seguindo a sugestão de Hájek (1997 e 2009), estou disposto a dizer que frequências relativas fornecem uma boa metodologia, mas uma análise insatisfatória do conceito de probabilidade física.

\section{REFERÊNCIAS:}

ACHINSTEIN, P. The Book of Evidence. Cambridge: Cambridge University Press, 2001.

BRADLEY, D. A Critical Introduction to Formal Epistemology. London: Bloomsbury, 2015.

CARNAP, R. Logical Foundations of Probability. 2nd ed. Chicago: The University of Chicago Press, 1962.

$\mathrm{CHURCH}, \mathrm{A}$. On the Concept of a Random Sequence. Bulletin of the American Mathematical Society, v. 46, n. 2, p. 130-135, 1940.

GILLIES, D. Philosophical Theories of Probability. New York: Routledge, 2000.

HACKING, I. The Logic of Statistical Inference. Cambridge: Cambridge University Press, 1965.

HÁJEK, A. Fifteen Arguments against Hypothetical Frequentism. Erkenntnis, v. 70, n. 2, p. 211-235, 2009. 
HÁJEK, A. Interpretations of Probability. IN: ZALTA, E. (Ed.). The Stanford Encyclopedia of Philosophy, 2012. Disponível em: <http://plato.stanford.edu/entries/probability-interpret/>. Último acesso: 20 de outubro de 2018.

HÁJEK, A. 'Mises Redux' - Redux: Fifteen Arguments against Finite Frequentism. Erkenntnis, v. 45, n. 1, p. 209-227, 1997.

HÁJEK, A. What Conditional Probability could not be? Synthese, v. 137, n. 3, p. 273323, 2003.

HÁJEK, A; HITCHCOCK, C. Probability for Everyone - even Philosophers. IN: HÁJEK, A; HITCHCOCK, C (Eds.). The Oxford Handbook of Probability and Philosophy. Oxford: Oxford University Press, 2016, p. 5-30.

HANDFIELD, T. A Philosophical Guide to Chance: Physical Probability. Cambridge: Cambridge University Press, 2012.

KOLMOGOROV, A. N. Foundations of the Theory of Probability. 2nd ed. New York: Chelsea Publishing Company.

KYBURG JR, H. E.; TENG, C. M. Uncertain Inference. Cambridge: Cambridge University Press, 2001.

LA CAZE, A. Frequentism. IN: HÁJEK, A; HITCHCOCK, C (Eds.). The Oxford Handbook of Probability and Philosophy. Oxford: Oxford University Press, 2016, p. 341-359.

LAPLACE, P. S. A Philosophical Essay on Probabilities. New York: Dover, 1951.

MELLOR, D. H. Probability: A Philosophical Introduction. London: Routledge, 2005.

RAMSEY, F. P. Truth and Probability. IN: RAMSEY, F. P.; BRAITHWAITE, R. B. (Eds.). The Foundations of Mathematics and Other Logical Essays. London: Routledge, 1950, p. 156-198.

REICHENBACH, $\mathrm{H}$. The Theory of Probability. Berkeley, CA: University of California Press, 1949.

SALMON, W. C. The Foundations of Scientific Inference. Pittsburgh: University of Pittsburgh, 1966.

VENN, J. The Logic of Chance. 3rd ed. London: Macmillan, 1888.

VON MISES, R. Probability, Statistics and Truth. New York: Macmillan, 1957. 